\title{
Real-time cloud system for managing blood units and convalescent plasma for COVID-19 patients
}

\author{
Dhuha Basheer Abdullah ${ }^{1}$, Mohammed Dherar Younus ${ }^{2}$ \\ ${ }^{1}$ Computer Sciences and Mathematics College, Mosul University, Iraq \\ ${ }^{2}$ Computer Sciences Department, Computer Sciences and Mathematics College, Mosul University, Iraq
}

\begin{abstract}
Article Info
Article history:

Received Sep 8, 2020

Revised Jan 11, 2021

Accepted Jan 21, 2021

\section{Keywords:}

Blood bank system

Blood units management

Cloud computing

COVID-19

Healthcare system

Real-time systems

ABSTRACT

In health care systems, blood management services are essential to saving lives. In such systems, when a unit of blood is required, if the system is not able to provide it on time, sometimes this may lead to patient death, especially in critical cases. Unfortunately, even if the required blood unit is available within the system, contradictions may occur and the required blood unit may not be allocated to critical cases on time, due to the allocation of these units to lower priority cases or due to the isolated operate of blood banks within these systems. So, to overcome these obstacles, we proposed a real-time system on a cloud, to managing blood units within the whole health care system. This system will allocate blood units depends on the deadline and the severity of the case that needs blood, in addition to the types, quantities, and position of available blood units. Where, this system eliminated the need for human intervention in managing blood units, in addition to offering the ability to easily develop the system to deal with new urgent requirements, which need new methods of managing blood units; as is happening today with the COVID-19 epidemic. This system increases the performance, transparency, reliability, and accuracy of blood unit management operations while reducing the required cost and effort.
\end{abstract}

This is an open access article under the CC BY-SA license.

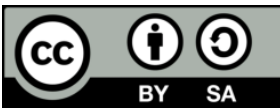

Corresponding Author:

Mohammed Dherar Younus

Department of Computer Sciences

Mosul University

Mosul, Iraq

Email: mohammedalkazal@uomosul.edu.iq

\section{INTRODUCTION}

In healthcare systems, resources are usually sensitive and scarce, so these resources must be managed effectively, accurately, and transparently. One of the most important of these resources is blood units (whole blood, red blood cells, double red, plasma, and platelet separation) [1]. Where the process of managing blood units is one of the most sensitive and difficult challenges in health care systems and the responsibility for this process rests with the blood bank systems [2]. Where banks must provide appropriate blood units to patients in a timely manner, and in the event of any delay or error in the process of providing the blood unit, this will lead to catastrophic results that may cause the loss of the patient's life [3]. This process is also more important in emergency situations, disasters, and the spread of epidemics; as happens these days in light of the coronavirus disease (Covid-19) epidemic [4].

The main problems in the operations of managing blood units are that most of the current traditional health care systems such as the health care system of the city of Mosul (Nineveh Governorate in Iraq) contain many blood banks, donation camps, and blood allocation centers that operate independently and isolated 
from each other [5]. Where, these systems do not provide blood banks with direct communication capabilities with each other, or with communication capabilities with donors and beneficiaries. Also, the decision-making processes to manage blood units in these systems depend entirely on human intervention. This is done by using paper documents and relying on the traditional method of allocation and direct delivery of blood units according to the first-in-first-serve algorithm (FIFS) [6]. This traditional method suffers from many problems and obstacles, as critical cases sometimes do not get their needs from blood units because they are allocated to the lesser priority cases that arrived earlier, in addition to the possibility of human errors and the complexities of managing paper files [7]. To reduce the impact of these problems, blood banks rely on a policy of maintaining the minimum safe storage and long-term storage of blood units, and here each blood bank needs special equipment that is financially expensive to store blood units in the long term. Also, blood banks rely on their operations to compensate for the shortage of blood units due to consumption, on cases of personal motivated donation or group donation campaigns. Unfortunately, these solutions may fill the shortage in the quantities of blood units, but the types of new blood units that enter the system from these methods often do not correspond to the needs of the system, because the demand and donation processes are separate and isolated. Here in many cases, providing the appropriate unit of blood becomes a patient's problem [8].

There is no doubt that health care systems benefit greatly from the technological developments that have been achieved in recent years in the development of blood unit management systems. Where, today some researchers focus on their work on building computerized systems to manage blood units with better performance, effectiveness, and cost. These new systems often rely on cloud computing to provide databases to save Information of donors, blood units, and beneficiaries, in addition to the use of smart device applications to facilitate access to the database by users [9]. These research and systems contributed to discovering and solving many problems and obstacles that were previously faced by the process of managing blood units [10]. Where, many researchers in blood bank management systems found that there is no unified approved system for donating in blood banks, and hospitals still use paper or Excel sheets in the computer to save data [11]. Also, they found that the only way of communication between blood banks, donors, and patients in emergency cases is done by a phone call or SMS [12]. So they focused on the possibility of implementing systems that provide a complete network for blood banks with mobile applications, to form a communication path between the blood bank and the donor and the patients, and anyone can download this mobile application from the App store, also there will be a website for people who use a computer. These systems somewhat solve the problem of the lack of communication between blood banks, donors, and patients [13].

Other researchers worked on developing an integrated framework for all relevant and isolated online subsystems for the blood management system .they propose a data warehouse (DW) as part of the integrated framework a central database for storing historical blood donation data for analytical processing [14]. They also try in their work present a new standard for citizenship, which is a system of scoring points for good deeds for citizens called it philanthropy score (PS), this system will enable the agencies responsible for the healthcare systems making a blood donation camp based on analytical reports from DW. Also, the declared PS points for the citizens will be an additional incentive for them to donate blood [15]. Some other researches focus on the problem when the blood unit is required in a hospital, and the hospital cannot be provided in time, which will cause unpleasant things. This is despite the fact that suitable donor data is often available in the hospital's database, but the donor does not know this, nor does he know the location of the blood bank that needs the donation. To solve this problem, there will be an Android application offer blood bank maps and establish communication between the hospital, receptor, donor, and blood bank [16]. In addition to that, some research worked on the idea of searching for the closest suitable donors in terms of location, to direct it to the blood bank that needs to donate. The methodology used to build such systems uses GPS services [17]. All that aims to generate full information about the donor and the organization related to blood donation, which will speed up the donation operation.

This increase in the use of smart devices, information technology, and networks in many aspects, especially health services caused radical changes in health care systems. The researches show that the widespread use of smart devices and other modern technologies can reduce doctor stresses, reduce health care costs, and speed the provision of patient care and easy access to patient medical information. This use of smart devices and information technology will not replace health personnel, but help them to provide better health care, by providing information more easily [2]. Also, researchers are starting to study the effects of the increasing use of digital sensors in smart devices and modern medical devices, with cloud computing and the internet of things (IoT) technologies. Where, this enabled developers to use these new techniques to build applications that work in real-time disease monitoring and early warning. The researchers aim to identify convergence opportunities in the medical field through the applications of smart devices, enabled by recent technological developments in medical devices and the use of methods and technologies of cloud 
environment platforms, through which patient data can be provided in real-time to doctors in remote locations [18]. These techniques are also used in building real-time systems to monitor stored blood units in terms of location, quality of storage process, and validity of its use. These developments have greatly improved the performance of blood units' management systems in some aspects, but it has not solved all major problems. Where, it is noted that most of these systems focus on offering the related data to the health care staff in easy and fast, in addition to providing the capabilities of analyzing and sorting data while reducing costs and facilitating documentation and archiving. All of this will enable the blood managing administrator to manage blood units more efficiently, accurately, and in a more comprehensive manner. Also, these systems provided communication possibilities between those in need of blood units and donors within the health care system [10].

Unfortunately, these systems still depend on human intervention in the blood unit management and especially in the decision made of blood unit allocation, which still a major reason for the poor performance of the blood unit management process and a source of errors and obstacles. This has negatively affected the health care services provided to patients and sometimes it leads to the loss of patients' lives. This appears clearly when the geographical or service coverage covered by these systems expands, or when disasters occur, or in cases of epidemics, which it leads to an increase in the need for real-time response, with the increase in the loads on the system and the blood managing administrator [9]. Also, these systems still lack full communication capabilities and integration in working between their parts, as every blood bank, donation camp, or hospital within the system is still operating and it takes its decisions to manage blood units in large percentage isolation from the rest of the system.

Therefore, the need arose to build new systems for managing blood units that provide all previous services and benefits and providing more communication and integration between all parties in the process. In addition to reducing dependence on human intervention, where this new system should have the ability to respond and make decisions related to the management of blood units in a timely manner for all parties involved in the process within the healthcare system; including dealing with allocation requests, transfer of blood units, and generating specific pre-emptive requests for blood donation. Moreover, this system must also offer the ability to accommodate any change in the current blood unit managing cases as well as the new cases that may appear in the future, and this is what we work on it in this paper.

\section{THE PROPOSED METHOD}

The decision-making process to manage blood units within the healthcare system is one of the most complex and sensitive due to its direct impact on human life. The previous and current blood unit management systems suffer from several problems related to each other and lead to limited performance and capabilities of these systems, where the most important of which is relying on human intervention in making decisions about managing blood units. The difficulty lies in eliminating the reliance on human intervention in making decisions, in the diversity and complexity of these decisions, in addition to the complexity and overlapping of data, conditions, and rules upon which these decisions depend on; all that may change from one case to another [19]. As the operations of managing blood units depend on the contexts approved by the health care systems, which depend on the type and conditions of disease cases that are dealt with in addition to the type and quantity of available blood units or the extent of their availability. Also, these contexts may change completely in emergencies, disasters, and epidemics [20]. For example, the significant influence of the level of oxygen in the blood on the decision to allocate plasma units for treatment as a treatment for severe COVID-19 patients [21]. All this complicates and makes it difficult to build systems to manage blood units that eliminate the need for human intervention in decision-making as well as accommodate all current and future situations.

The method that we propose in this paper as a solution to this problem is to build a central system that is efficient, flexible, highly reliable, easy-to-use, and has high performance. This proposed system must have the potential to make decisions for managing blood units based on available data. The proposed system should also provide the possibility of updating and improvement to increase the efficiency of blood unit management operations for current cases while providing the possibility of development and addition to accommodate the operations of managing new cases. In order to solve the problem of isolating the parties and institutions involved in the process of managing blood units and increase the integration and comprehensiveness of decisions, the system must be centralized and comprehensive. The proposed system should also benefit from the results of previous research and the ideas and principles of the systems it proposes. Whereas, the proposed system, given the sensitivity of blood unit management processes, should be highly reliable and provide capabilities to bypass technical failures without affecting blood unit management operations. In addition, it would be good for the beneficiaries to access and use this proposed system in an easy and reliable way. So, if all the previous points are available in the proposed system, it will

Real-time cloud system for managing blood units and convalescent plasma for... (Dhuha Basheer Abdulla) 
inevitably increase the efficiency, transparency, and accuracy of the operations of managing blood units significantly and make the possibility of human errors in the process of managing blood units almost nonexistent. Also, in addition to all of the above, it will significantly reduce your required cost and effort.

\section{RESEARCH METHOD}

To meet the points and needs mentioned in the proposed method paragraph in our proposed system, we relied on the use of a hybrid of real-time algorithms, in order to ensure that efficient management decisions are made in a timely manner. We have also taken into account when we designed and implemented the tasks and operations in the system that each one of them must be an independent entity and isolated from other entities, which will make the process of developing these entities or adding other entities to provide more services or solve future problems is very easy. We also used principles and algorithms for multi-source supply chains, consumers, and competent to deal with limited and sensitive resources. As a medium for the system to work, we used the platform provided by the public cloud environment, to make the system more reliable and reliable in addition to providing easy and fast access to all users as well as reducing the cost of construction and maintenance [22]. We also adopted in the design and implementation of system models and data stores to be isolated and independent to facilitate the modernization and development processes, in addition to overcoming the big data problems associated with such systems [23].

\subsection{Proposed system}

In this paper, we propose a cloud-based, real-time system that centrally manages blood units within a range of blood banks, hospitals, and donation centers. This system has the ability to make decisions to manage blood units without any human intervention while providing the ability to add and develop the services it provides in a flexible and easy way. This is what distinguishes this system from the rest of the current traditional systems. The design of this system takes into account the great impact of its work on human life in most cases, as it can be classified as a hard real-time system in general [24]. The main part of this system is the main management server, which is responsible for making management decisions, which will depend on a hybrid of real-time algorithms. This hybrid of algorithms is divided into two main parts, the first part uses the polling server algorithm, and it is responsible for organizing the work of periodic tasks and responses to the entire system. Among these periodic tasks in the system is to keep track of the connection status of the local backup and support systems, check the expiration date of blood units, analyze the status of blood stocks in the system, run the scheduling process for periodic blood requests (thalassemia, spleen atrophy, and kidney failure), in addition to organizing the work periods of the second part of the system algorithms. The second part will be responsible for responding to aperiodic events, and the most important of these events are the processes of requesting the allocation of blood units.

The second part uses the earliest deadline first (EDF) algorithm, in addition to the priority principle to distinguish between the different types of blood unit requests based on the severity of the case. Where, the search for blood units appropriate for the requests will be done at the local and then on global levels. Where the system will start searching for the appropriate unit in the current blood bank stock, otherwise it will move to search for the appropriate unit within the stocks of the rest blood banks from closest to farther away. Moreover, these algorithms will use the time between allocating a unit of blood and it is actual consumed by the patient, in the reallocation of the blood units to allocation requests in a better manner. This gives the allocation requests of the critical cases the possibility of extracting the allocated blood units from other lower priority cases, in the absence of appropriate unallocated units in the system. Here, the algorithms of the second part will use the data and information in the main data store as determinants to make decisions related to the allocation of blood units.

Also, the proposed system contains the main data store located in the cloud environment, in addition to a set of local data stores distributed to blood banks and hospitals, which act as local backup databases. Therefore, we relied on building databases on SQL database, due to their ability to create and organize large and complex databases with precision, ease, and flexibility. On the one hand, using SQL to build databases in the system made it easier to link and overlap the master database and backup databases, making switching and moving between the backup and backup system to the cloud host in an emergency easier. In addition to, provide access to more than one user to the databases at the same time without problems. Whereas, the main data store will contain all data and information such as details about the types, quantities, and locations of the blood unit storage in addition to donor data and allocation requests. It will also facilitate the process of entering the information sent by registered users.

\subsection{Proposed system modules}

The architecture of the proposed system consists of three main modules as shown in Figure 1.

Int J Elec \& Comp Eng, Vol. 11, No. 4, August 2021 : 3593 - 3600 


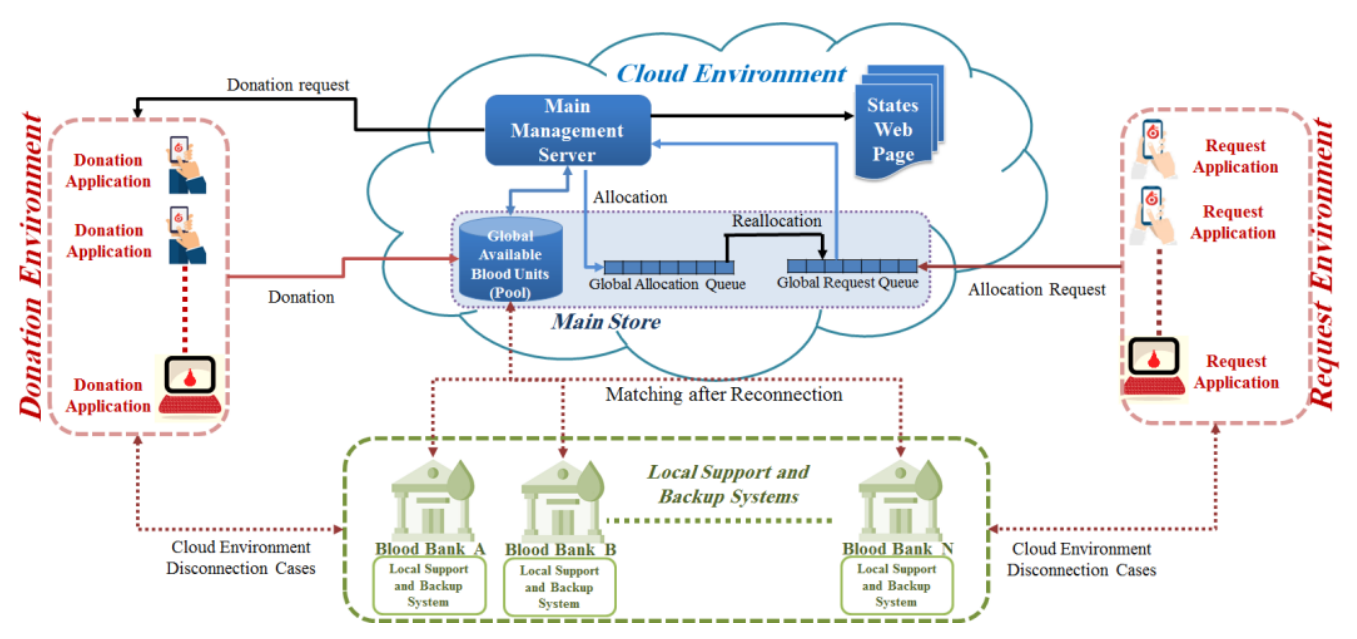

Figure 1. Proposed system modules

\subsubsection{Cloud environments}

This component includes the main management server and the main data store. The main management server is the heart of the system and the most important part of it, which is what, distinguishes the system from the rest of the previous systems. It contains real-time algorithms for allocating, managing, and distributing blood units according to the precedence of incoming requests and depending on the resources available locally or in other blood banks in the system. All user authentications are only made by the server. The main data store contains the main database of blood units and donors and blood units request queue for all blood banks. The main data store enables the rest models in the system to access the database and update it easily and without conflict. The cloud environment also contains authentication operations, which allow only registered users to access the system. In addition, the cloud environment will contain the status web page, which displays general information about the level of storage and needs for blood units to the public and the users concerned. Here, we relied on the services provided by Microsoft (Azure) for the great capabilities it provides, especially in addressing server problems during times of increased usage pressure and the expansion and complexity of databases [25]. It is also the most suitable and closest option to the systems and applications already in use and the most popular choice for users [26].

\subsubsection{Smart devices application}

By using these components, the user can quickly and easily access the database. We have two types of applications; the first one provides the donation environment, which works as a user interface for blood donation camps to enter donor information, the location of the donation, and the type of blood units entered into the system. The application also provides the ability to receive messages addressed from the server, which contain information about the blood units required to be provided to meet the needs. The second application provides the requests environment which enables the authorized health staff to create requests for blood units through an interface to enter the required blood unit information, the patient's condition, and the location of the case. After allocating the blood unit, the applicant receives the allocation message from the server through the same application.

\subsubsection{Support and backup systems}

Due to the sensitivity of the proposed system and its direct impact on patients' lives, each local blood bank will have a local data store and a local management server. The local data store will act as a secure backup system for the information of the main data store. Also, it can be used within the local region in cases of communication problems with cloud environments or emergency breakdowns. The local server will, in normal circumstances, provide a user interface that enables the responsible employee to record the operations on the units such as donation, allocation requests, and delivery. In addition to providing the ability to managing the registered users' information and authentication as well as displaying the allocating reports of the local region. In abnormal circumstances, such as disconnection from the cloud environments or emergency breakdowns, the local management services will provide the same capabilities as the main server within the local region. Here the support and backup system will save all information about the operations within the local region in the local data store until returned the connection with cloud environments, in order to be loaded in the main data store. 
The system design provides the possibility to implement this component in a number of ways, including the use of a personal computer as a local management server with a local data store within the same device. Here, in emergency circumstances, the local backup and storage system will not provide services for smart device applications, so all personalization and donation requests will be executed in the traditional manual way. The design also provides the ability to use the private cloud in implementing this component. Here the applications of smart devices within the local domain will be linked to the management server and data store within the private local cloud, which in turn will be linked to the public cloud, as well as the ability to build a broader hierarchical system, through the use of the public cloud in implementing this component. When we need to expand the current system to include wider geographical areas with a greater number of blood banks, or to develop it to reduce the workload and increase its reliability and performance.

\subsection{Management of convalescent plasma for severe COVID-19 patients}

Recently, due to the conditions of the COVID-19 pandemic, a need has emerged to assist doctors in providing convalescent plasma from people who have recently recovered from the coronavirus disease (COVID-19) as a therapy for severe COVID-19 patients. This was a real test of the proposed system's flexibility and scalability to accommodate and service new and exceptional blood unit management cases. Here we have added a new service to the proposed system that enables it to manage convalescence plasma for severe COVID-19 patients. We added new labels within the databases and the data stores to represent the percentage value of oxygen in the blood of severe COVID-19 patients as a priority degree and the recovery date for the donor of convalescence plasma as a preference degree. In the management servers, we added a new function that allocates convalescence plasma units based on the percentage of oxygen in the blood of COVID-19 patients in addition to the type of plasma unit required and the donors recovery date.

On the other hand, we have updated smart device applications to enable application users to benefit from this new service in addition to previous services. Where, in this new update of the smart device's application, users can choose between requesting for blood unit allocation and requesting for convalescence plasma unit allocation with the determination of the percentage of oxygen in the patient's blood. At the same time, it will enable the donation application user to choose between registering donating blood units and donating units of convalescence plasma with specifying the date of donor recovery. And this update will be available to users easily on the approved application store.

\section{RESULTS AND DISCUSSION}

The proposed system generally provides better performance than traditional systems, as well as is characterized by ease of use with low required cost and effort. In the testing of the performance and efficiency of the proposed system, we used data from the Central Blood Bank in the city of Mosul. This data contained detailed information about all blood units' types, donors, allocation requests, and allocation operations by the traditional system for a period of six months in five local blood banks. Figure 2 shows a comparison between the performance of the conventional system and the proposed system based on the percentage of blood unit allocation failure in the two systems. Where, we note that the percentage of blood unit allocation failure decreased significantly when we use the proposed system. Moreover, in the proposed system, the decrease in the blood unit allocation failure percentage for critical cases was greater than the decrease in normal cases. These percentages continued to decrease with the increase in the number of blood banks (BB) within the proposed system. In addition, the proposed system succeeded in accommodating all pre-existing blood unit allocation cases, and it also succeeded in accommodating new cases by providing updates and adding capabilities. As we have done, when the need to add a new part to the system appeared to allocated convalescent plasma to severe COVID-19 patients. This success was not only in accommodating this process but in increasing the rate of allocation of convalescent plasma to severe COVID-19 patients, based on the percentage of oxygen in the patient blood.

The use of the proposed system also reduced reliance on random donation campaigns to compensate for the shortage in blood units, and thus provided more opportunities for targeted donation cases. Whereas, if donating a unit of blood is necessary anywhere, the system will direct requests to a number of the closest potential donors to fill this shortage. This also reduced the need for long-term storage of blood units and provided more renewable and non-stored blood units for long periods because the ability to supply new blood units became constantly possible. Moreover, this provided a stable basis for the communication process between hospital staff, blood banks, donors, and patients.

The local support and backup systems also contributed greatly to increasing the reliability of the system, as it helped a lot in overcoming technical problems and Internet service. The use of smart device applications by registered users and blood banks registered in the system speeds up and simplifies the processes of entering and arranging blood units in the proposed system. These applications also enabled users 
to use their smart devices in the processes of requesting and entering blood units in the system, where they can obtain these applications and their updates through the approved application store. Where, for the registered users, they should only use the appropriate application to donate or request and then enter the required information about the type of blood unit and the type of case, and the system will be responsible for providing the appropriate unit of blood in the most appropriate time possible.

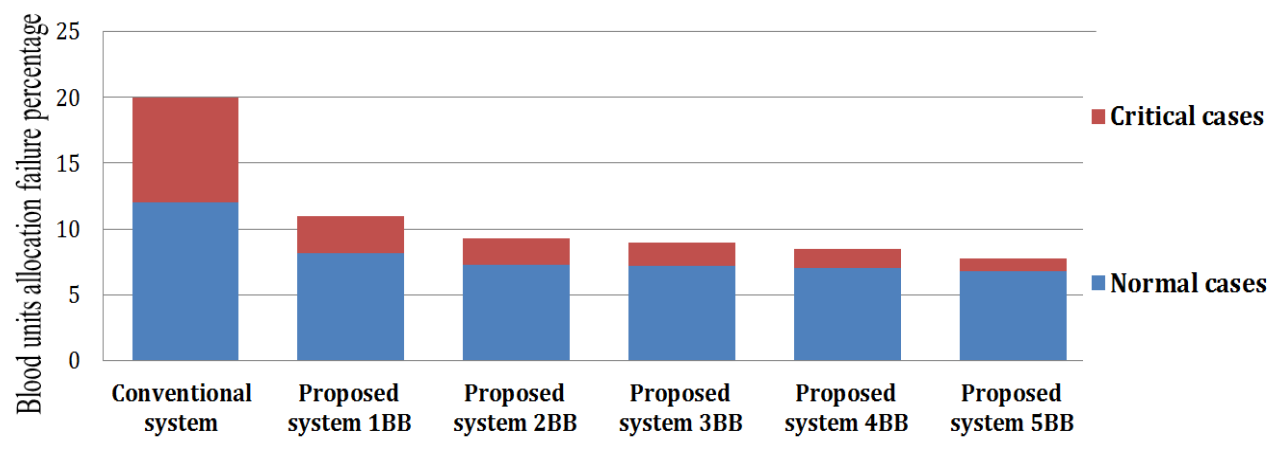

Figure 2. Comparison between the conventional and the proposed system in the allocation failure percentage

\section{CONCLUSION}

The proposed system provided unified and centralized management of blood units within all blood banks and hospitals within the health system, and thus the process of allocating the required blood units in a timely manner became more effective, accurate, and transparent than before. Also, it is eliminated the need for human intervention in making decisions about managing blood units, resulting in increased performance while reducing process errors and wastes. Where, it becomes possible for the patient to obtain the appropriate unit of blood from the nearest source within the health system that can provide it at the right time, instead of relying on local sources only. This has increased the performance of the system and the comprehensiveness of allocation processes. Moreover, the use of the proposed system will provide in the future a complete and detailed database for donating, ordering, assigning, and transferring blood units stored in the cloud environment. Where this data can be used as analytical and research materials to detect patterns of disease spread, cycles of high demand for blood units, accidents, and emergencies. This contributes to building systems that are more predictable for future blood unit needs. All that in addition to, possibility benefit in the future from the algorithms of artificial intelligence to develop system performance.

On the other hand, the three-modular architecture, in addition to building each management server to operate in a separate and independent manner; will provide possibilities for developing and expanding the system in the future to provide additional services or cover wider geographical areas in an easy and flexible way. This makes the proposed system a solid base for building broader and more comprehensive healthcare systems. Where it can deal with and contain exceptional circumstances by developing system services and adding new services. The proposed system services can also be developed to include the management of other medical services, such as medical diagnostic reports, disease reports, digital radiographs, and medicines. This will lead to providing greater opportunities for developing and improving health care services provided to beneficiaries.

\section{REFERENCES}

[1] M. A. M. Shoaib, "Hospital management, applied perspective, contemporary management, information systems, health economics systems and situational management systems," Part IX, ALMANAHL, 2014.

[2] S. M. Z. Rahma and A. M. Saleh, "Healthcare System Technology using Smart Phones and Web Apps (Case Study Iraqi Environment)," I. J. Engineering and Manufacturing, vol. 3, pp. 1-7, 2017.

[3] Zu-Jun M., Ke-Ming W., and Y. Dai, "An Emergency Blood Allocation Approach Considering Blood," Int J Disaster Risk Sci, vol. 10, pp. 74-88, Dec. 2018.

[4] B. L. Browna and J. McCulloughb, "Treatment for emerging viruses: Convalescent plasma and COVID-19," Transfusion and Apheresis Science, vol. 59, no. 3, 2020, Art. no. 102790.

[5] Mahmoud M. A., Ahmed A. H., and Nasir M. Y., "Knowledge, Attitude, and Practice (KAP) of Voluntary Blood Donation among Nurses in Mosul Teaching Hospital," Mosul Journal of Nursing, vol. 5, no. 1, pp. 24-32, 2017.

[6] T. H. Jenipha and R. Backiyalakshmi, "Android Blood Donor Life Saving Application in Cloud Computing," American Journal of Engineering Research (AJER), vol. 3, no. 2, pp. 105-108, 2014. 
[7] R. N. N. Staggers, "Health Informatics 2nd Edition an Interprofessional Approach," Elsevier, 2017.

[8] Patrick M. et al., "Patient Blood Management is Associated with a Substantial Reduction of R B C Utilization and Safe for Patient's Outcome a Prospective," Annals of Surgery, vol. 264, no. 2, pp. 203-211, 2016.

[9] A. M. Mostafa, A. E. Youssef, and G. Alshorbagy, "A Framwork for a Smart Socialblood Donaation System Basdon Mobile Cloud Computing," Health Informatics -- An International Journal (HIIJ), vol. 3, no. 4, pp. 1-10, Nov. 2014.

[10] Prajakta et al., "Online Healthcare System Using the Concept of Cloud Computing," International Journal of Innovaive Resarch in Electrical, Electronics, Instrumention and Control Enginering, vol. 3, no. 10, pp. 61-64, Oct. 2015.

[11] A. A. Kayode, A. E. Adeniyi, Roseline O., Ogundokun, and S. A. Ochigbo, "An Android based blood bank information retrieval system," Journal of Blood Medicine, pp. 119-125, Jul. 2019.

[12] A. Al-Kalbani, S. I. A. Kazmi, and J. Pandey, "IoT Based Smart Network for Blood Bank," 2018 7th International Conference on Reliability, Infocom Technologies and Optimization (Trends and Future Directions) (ICRITO), Noida, India, 2018, pp. 732-736.

[13] V.K. Tatikonda and H. El-Ocla, "BLOODR: blood donor and requester mobile application," mHealth, vol. 3, 2017.

[14] Shubham P. et al," "E-Blood Bank Application Using Cloud Computing," International Research Journal of Engineering and Technology (IRJET), vol. 5, no. 2, pp. 278-281, Feb. 2018.

[15] G. Maji, N. C. Debnath, and S. Sen, "Data Warehouse Based Analysis with integrated blood donation management system," 2018 IEEE 16th International Conference on Industrial Informatics (INDIN), Porto, Portugal, 2018, pp. 855-860.

[16] S. Yuan, S. Chang, K. Uyeno, G. Almquist, and S. Wang, "Blood donation mobile applications: are donors ready?," TRANSFUSION, vol. 56, pp. 614-622, 2016.

[17] P. Jagtap, M. Mandale, P. Mhaske, S. Vidhate, and S. S. Patil, "Implementation of Blood Donation Application Using Android Smartphone," 3Open Access International Journal of Science \& Engineering, vol. 3, no. 1, Mar. 2018.

[18] M. A. Kazancigil, "Innovations and Convergence in Mobile Medical Applications and Cloud-Based Hospital Information Systems for the Real-Time Monitoring of Patients and Early Warning of Diseases," IEEE World Congress on Services (SERVICES), 2019, pp. 301-307.

[19] B. Sharma, M. Ramkumar, N. Subramanian, and B. Malhotra, "Dynamic temporary blood facility locationallocationduring and post-disaster periods," Applications of or in Disaster Relief Operations, vol. 283, no. 5, pp. 705-736, Oct. 2017.

[20] B. Whitaker, S. Rajbhandary, S. Kleinman, A. Harris, and N. Kamani, "Trends in United States blood collection and transfusion: results," TRANSFUSION, vol. 56, no. 9, pp. 2173-2183, 2016.

[21] K. Duan et al., "Effectiveness of convalescent plasma therapy in severe COVID-19 patients," Proc Natl Acad Sci U $S$ A, vol. 117, no. 17, pp. 9490-9496, Apr. 2020.

[22] A. Youssef, "Cloud Service Providers: A Comparative Study," International Journal of Computer Applications \& Information Technology, vol. 5, no. 2, pp. 46-52, 2014.

[23] B. S. P. Mishra, H. Das, S. Dehuri, and A. K. Jagadev, "Cloud Computing for Optimization: Foundations, Applications, and Challenges," Springer, 2018.

[24] J. W. S. Lue, "Real Time System," Prentice Hall, 2000.

[25] P. Dutta and P. Dutta, "Comparative Study of Cloud Services Offered by Amazon, Microsoft \& Google," International Journal of Trend in Scientific Research and Development (IJTSRD), vol. 3, no. 3, pp. 981-985, 2019.

[26] M. Copeland, J. Soh, A. Puca, M. Manning, and D. Gollob, "Microsoft Azure: Planning, Deploying, and Managing Your Data Center in the Cloud," Apress, 2018.

\section{BIOGRAPHY OF AUTHORS}

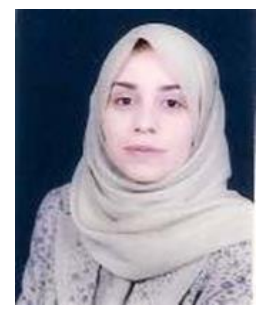

Dhuha Basheer Abdullah is the dean of Computers and Mathematics College, University of Mosul. She received her Ph.D. degree in computer sciences in 2004 in the specialty of computer architecture and operating systems. She supervised many Master and Ph.D. degree students in Operating System, Computer Architecture, Dataflow Machines, Mobile Computing, Real-Time Systems, Distributed Systems, Cloud Computing, and Big Data. She also leads and teaches modules at BSc, MSc, and Ph.D. levels in computer science. Also, she teaches many subjects for Ph.D. and master students.

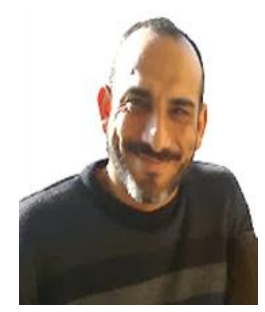

Mohammed Dherar Younus received the Bachelor of sciences from the Computer Sciences Department, Computer Sciences and Mathematics College, Mosul University, Iraq, in 2002. Currently, he is a master student in the Computer Sciences Department, Computer Sciences and Mathematics College, Mosul University, Iraq. He worked as a teaching assistant in the Department of Computer Engineering Technology at Al-Hadba'a University College NGI, Mosul, Iraq. 